\title{
Design of Narrowband Band Pass Filter using Open-loop Square Resonators with Loading Element
}

\author{
Venkata Varunbabu Mannam ${ }^{1}$ and Yedukondala Rao Veeranki ${ }^{*}$ \\ 'National Instruments, Bannerghatta Road, Bangalore - 560029, Karnataka, India; mannamvarun@gmail.com \\ 2Manipal Institute of Technology, Manipal - 576104, Karnataka, India; ykraoveeranki@gmail.com
}

\begin{abstract}
Objectives: In this paper we propose to design a compact, sharp-rejection narrowband BandPass Filter (BPF). Methods/Statistical Analysis: The behavior of the microstrip open-loop resonator is observed using full-wave electromagnetic simulations. By adding a loading element at the center of the open loop square resonator we are achieving two operating modes (even and odd modes) exist within a single resonator. Variation of parameters of the loading element leads to asymmetric frequency response used for filter design. The simulated results met all the design specifications of the given band pass filter. Findings: In the dual-mode open loop resonator the even and odd modes (currents are mutually exclusive in nature) do not couple each other and leads to a rejection point in the frequency, which is associated with the even mode. Multiple sections of resonator give the sharp rejection (high dynamic range) after the passband. Application/Improvements: The proposed band pass filter can be widely used in communication system and space technology, especially mobile and satellite communication due to its better performance and compactness.
\end{abstract}

Keywords: BandPass Filter, Dual-mode Resonator, Electromagnetic Coupling, Micro Strip Antennas, Strip Lines

\section{Introduction}

The electronic components with compact in size draws a lot of attention in the past few decades due to the very fast development of the mobile industry. Traditional high performance filters (like waveguide and dielectric resonator filters) are generally too heavy and bulky for applications like mounting on towers in base stations Similar behavior is observed in satellite applications where payload costs are increased a lot, and filters with better performance are typically required. Recently, the captured global market of mobile devices is pushing the needs beyond its limits. In recent portable products there is a limited use for bulk components with great performance. This is mainly due to the fact that majority of communication systems designed to wok below $6 \mathrm{GHz}$ frequency band.

At lower frequencies the resonators used are Bulkwave resonators, SAW (surface acoustic wave) filters and helical resonators. All these are used when small in size and low insertion loss are strongly demanded. Among the entire filter technologies microstrip remains popular because it is easy to integrate in the system, occupies small volume as well as its fabrication processes. Moreover, electronically tunable and reconfigurable filters, like the notch filters designed in ultra-wideband applications, uses surface mount varactors that are compatible with microstrip implementations ${ }^{2}$.The popular disadvantage of microstrip resonators is the low quality factors (Q-factor). However, for applications that need negligible insertion loss (like front ends of satellite receivers), or very narrow relative bandwidths, microstrip resonators provides great amount of quality factort.

By the way, the compactness in the microstrip component design leads to reduce the size of the required cooling system in the applications leads to over-all transceivers that are smaller than traditional transceivers, which usesdielectric resonator filters or waveguides ${ }^{1,3}$. The more information about band pass filter is referred from the references ${ }^{4-8}$. 


\section{Proposed Design}

The microstrip open loop resonator is animportant key structure for bandpass filter applications due to its crosscoupling nature, smallin size, of approximately $\frac{\lambda}{-} \mathrm{by}_{\frac{-}{2}}^{\lambda}$, and versatility9-12. The general structures of the square open loop resonators are shown in Figure 1.

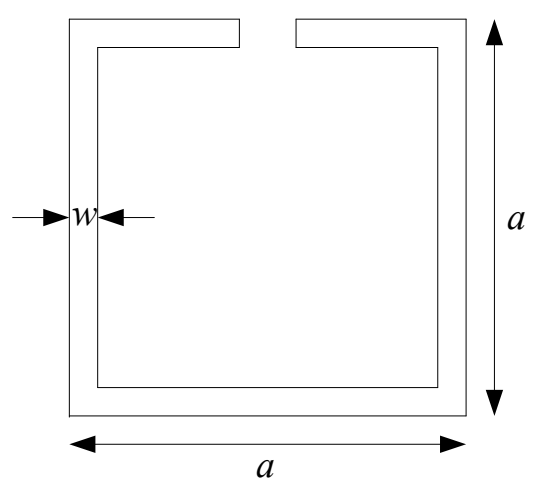

(a)

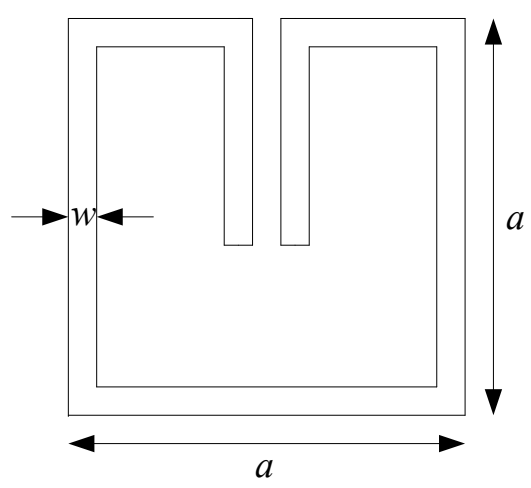

(b)

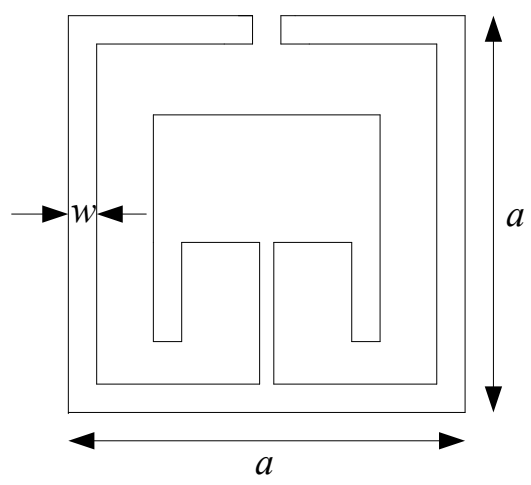

(c)

Figure 1. Square Open Loop Resonator (SOLR) and some miniaturization techniques. (a) Conventional SOLR (b) Folded arms SOLR (c) Dual mode SOLR.
The innovation of the wrapping arms (in a folded manner) square open loop resonator of Figure 1can be used as miniaturized hairpin resonators 1 . In the case of the wrapping arms resonator the size is further reduced more than what we thought due to the interesting phenomena of coupling between the arms upto $45 \%^{13}$. The wrapping arms structure can be designed with lower characteristic impedance in order to design the model with compact sizen. The resonator shown in Figure 2 works in a different way; it has two independent operating modes, and the electro-magnetic coupling between them can be modified by the parameters of the loading element. This new design of the resonator providing compact size model with reduction of $50 \%$. The method we proposed in this research is the square open loop resonator with a loading element at the center of the resonator as shown in Figure 1.

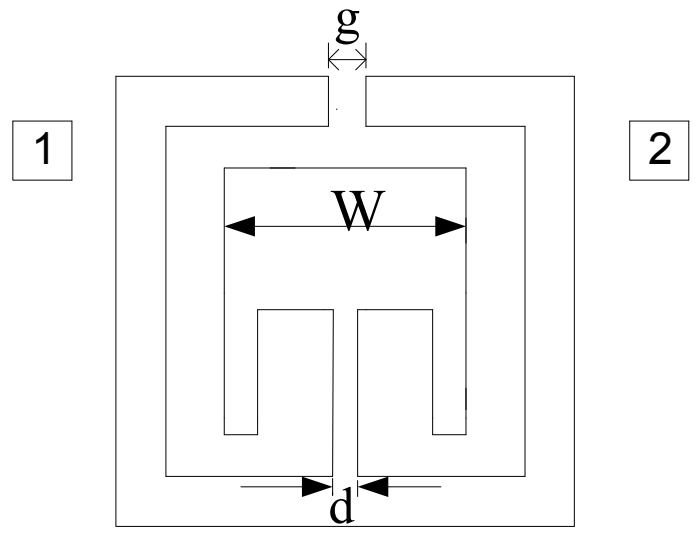

Figure 2. Dual mode open-loop square resonator.

Each single-mode resonator is equal to an LC coupled circuit, which produces a pole (transmission zero) at the resonance frequency. But in order to design a filter with sharp rejection number of single-mode resonators are to be connected in cascade structures and this leads to increase in size of the filter. Each Dual-mode open loop resonator (proposed design) is a doubly tuned LC resonant circuit. The number of resonators required for a given degree of filter is reduces by half because of the dual-mode behavior. The proposed design has the same size as single mode resonator with the help of loading element leads to compact in size. A loading element with a variable parameter ' $\mathrm{W}$ ' is placedat the center of the resonator. 


\section{Characteristics}

Change of width of loading element ' $\mathrm{W}$ ' leads to mode splitting. Two modes exist odd mode and even mode. The fixed frequency component of the band-pass filter by adjusting of width of loading element is called odd-mode. The variable frequency component by varying of width of loading element is called even-mode.

Here the effect of the loading element is shown in Figure 2 with different ' $W$ ' values on the microstrip open-loop square resonator on TMM10 substrate having dielectric constant $\left(\varepsilon_{1} r\right)$ of 10.8 with substrate height of $1.27 \mathrm{~mm}$.

Odd mode exhibits the same characteristics that of single mode resonator, since in the odd mode at the tapping point its short circuit. So the loading element does not contribute any coupling here. Hence the odd mode resonance frequency is dependent only on square open-loop parameters (i.e. length of resonator (a), width of resonator (w) and gap of open loop (g)). But in the even mode it causes the resonant frequency with varying the width of loading element ('W'), and width of tapping element ('d'). Figure 3-5 shows the variation of even mode resonant frequency with 'W'and 'd'.

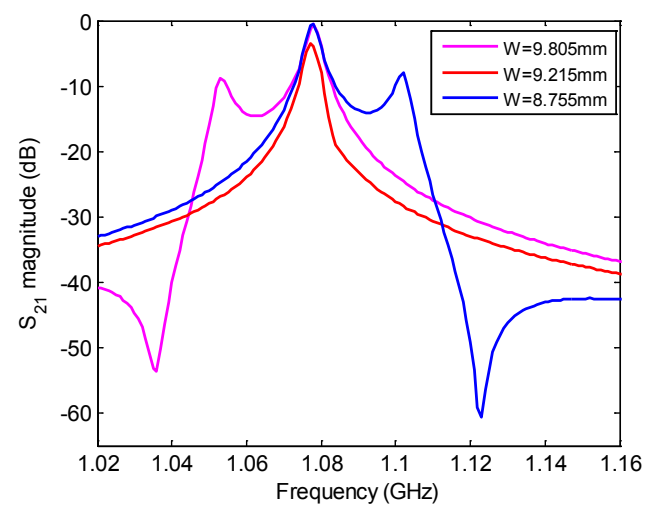

Figure 3. Modal-resonant characteristics of the proposed dual-mode microstrip open-loop resonator for $\mathrm{g}=0.9 \mathrm{~mm}$ and $\mathrm{d}=1.1 \mathrm{~mm}$.

To excite the resonator, weakly coupled ports are used. For a single value of ' $W$ ' the two modes exhibit same resonance frequency. As ' $W$ ' increases/decreases, the two modes split. Smaller'W', shifts resonance frequency of even mode to right. Larger ' $W$ ', shifts resonance frequency of even mode to left. Due to short circuit at tapped point no charge or current in the odd mode case in the loading element, and maximum current in the even mode case in loading element. There is a finite pole (transmission zero) in the insertion loss graph as the modes diverges. The finite pole is on right side if variable frequency component is more than fixed frequency component and on left side if fixed frequency component is more than variable frequency component.

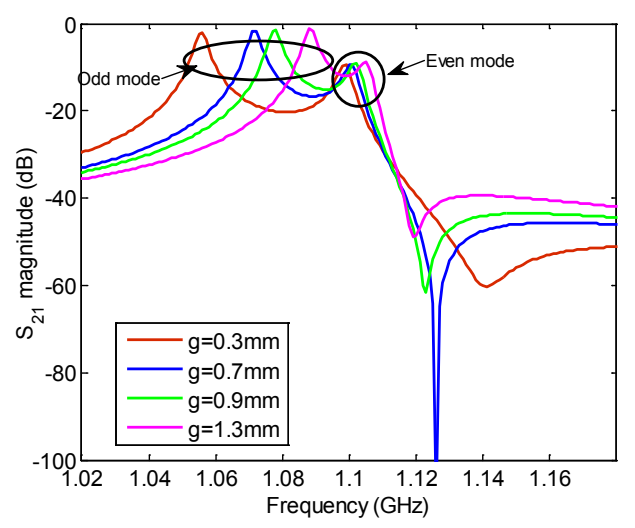

Figure 4. g-dependence of modal resonant characteristics of the proposed dual-mode microstrip open-loop resonator for $\mathrm{W}=8.755 \mathrm{~mm}$ and $\mathrm{d}=1.1 \mathrm{~mm}$.

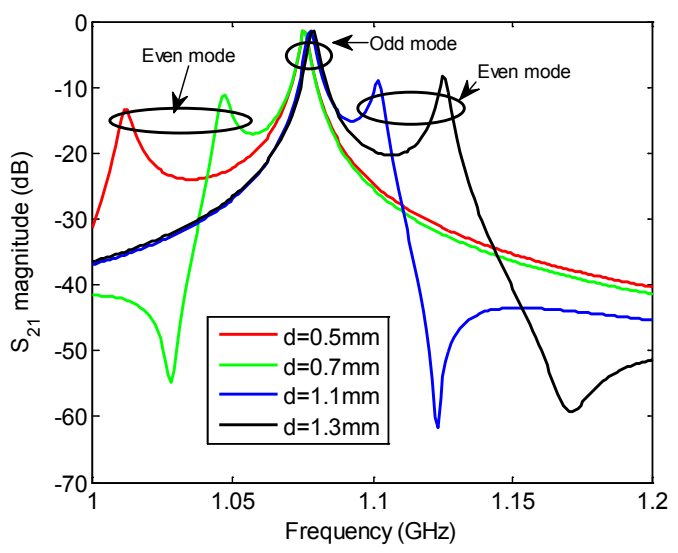

Figure 5. d- dependence of modal resonant characteristics of the proposed dual-mode microstrip open-loop resonator for $\mathrm{W}=8.755 \mathrm{~mm}$ and $\mathrm{g}=0.9 \mathrm{~mm}$.

\section{Deign Procedure for Open- loop Resonator}

The key parameters of the resonator:

The main parameters are length of resonator (a), 


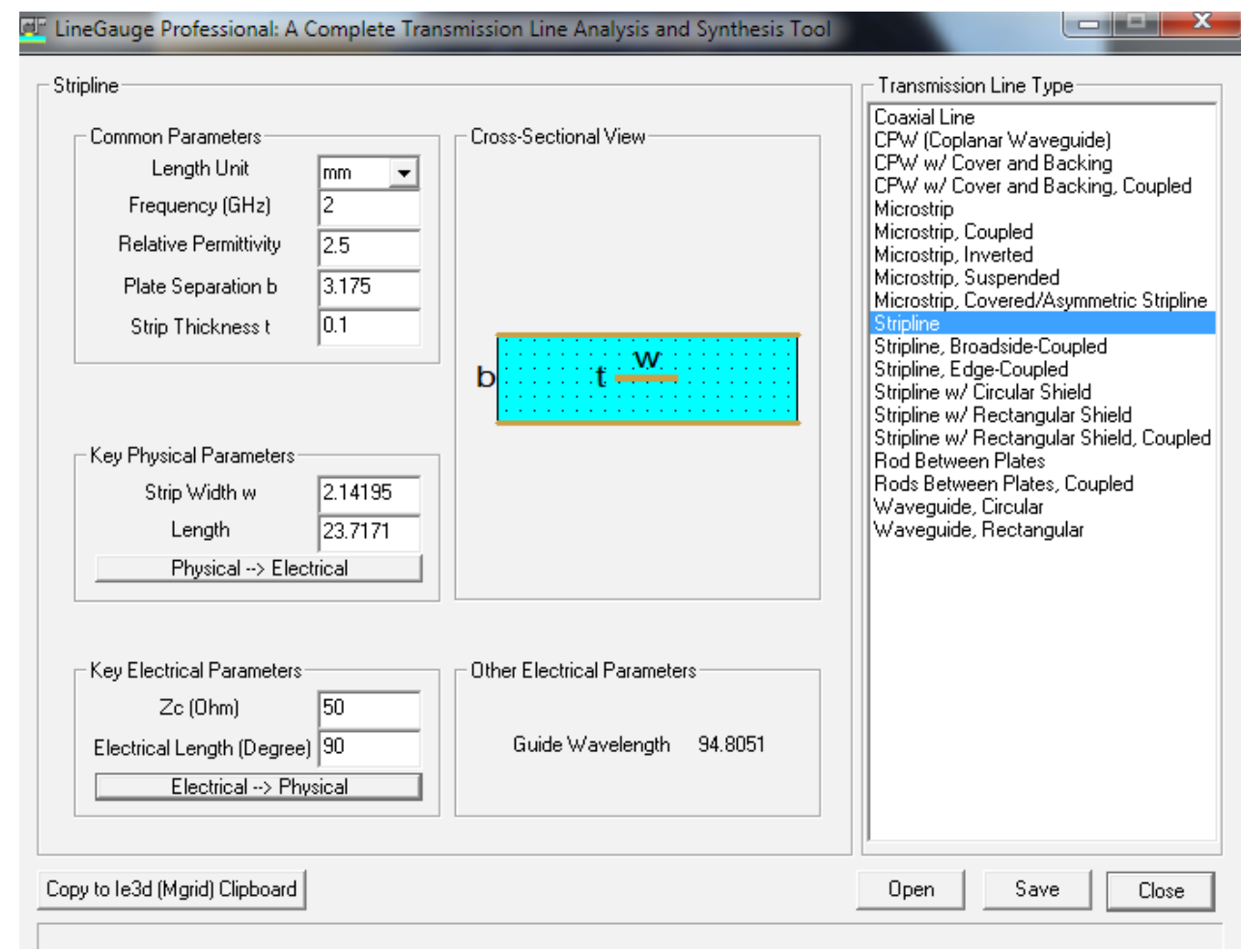

Figure 6. Length calculation of open-loop resonator from line guage.

width of resonator (w), gap of the resonator (g). Since it's an open-loop square resonator length and width of the resonator are almost equal.

\subsection{Length}

From the substrate details (dielectric constant, loss tangent, height of the substrate) and frequency of operation and the configuration (either microstripline or stripline) we can find the length of resonator (since it is a half wave length resonator), and width of resonator by the characteristic impedance of the resonator. From the Figure 6 the length of the resonator which is half wave length resonator $(47.40255 \mathrm{~mm})$. So the side of resonator is $(1 / 4$ th of the resonator length $(11.85 \mathrm{~mm}))$.

\subsection{Width of resonator}

From Figure 7 it is clear that there is relation of between the insertion loss $\left(\mathrm{S}_{21}\right)$ and bandwidth (BW) of the resonator. The requirements of low insertion loss and narrow band width of filter to design is a challenge here. So proper width of the resonator is chosen so that for the narrowband application along with low insertion loss characteristics.

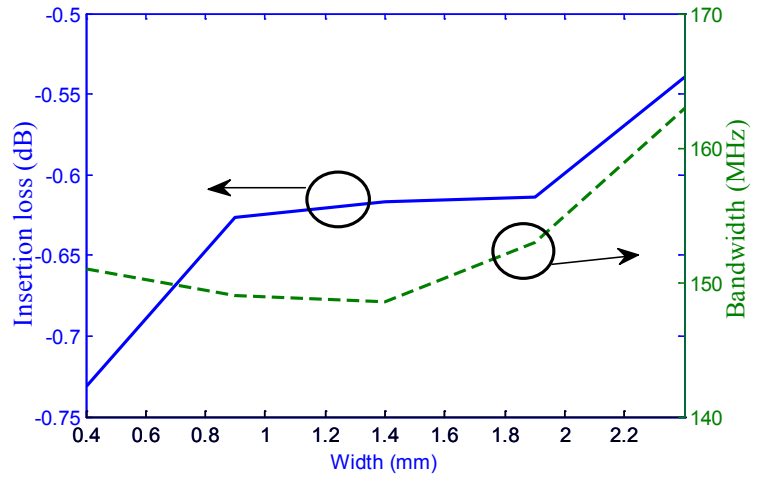

Figure 7. Width of the open-loop resonator vs. insertion loss and bandwidth.

\subsection{Gap}

Now the open loop gap is going to choose so that oddmode resonance frequency is going to exactly coincide with center frequency for the band pass filter design. Figure 8 shows the microstrip width of the loading element vs. insertion loss at odd and even-mode frequencies and Figure 9 shows the microstrip width of the loading element vs. resonant frequencies variations of dual-mode open-loop square resonator. 


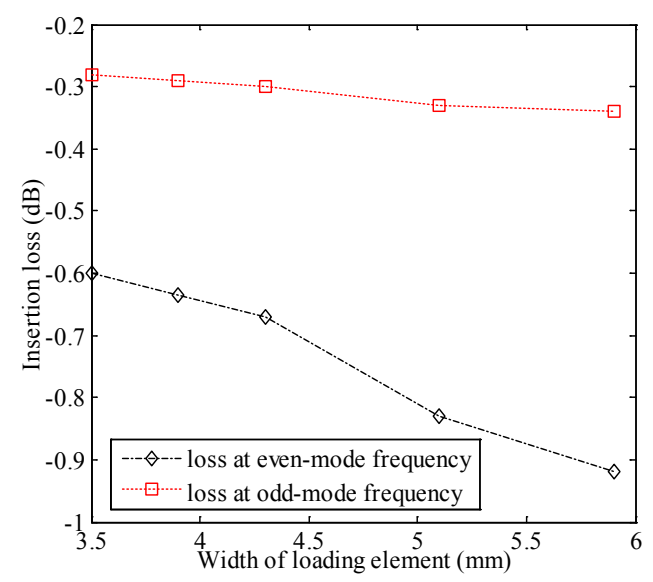

Figure 8. Width of the loading element vs. insertion loss at odd and even-mode frequencies.

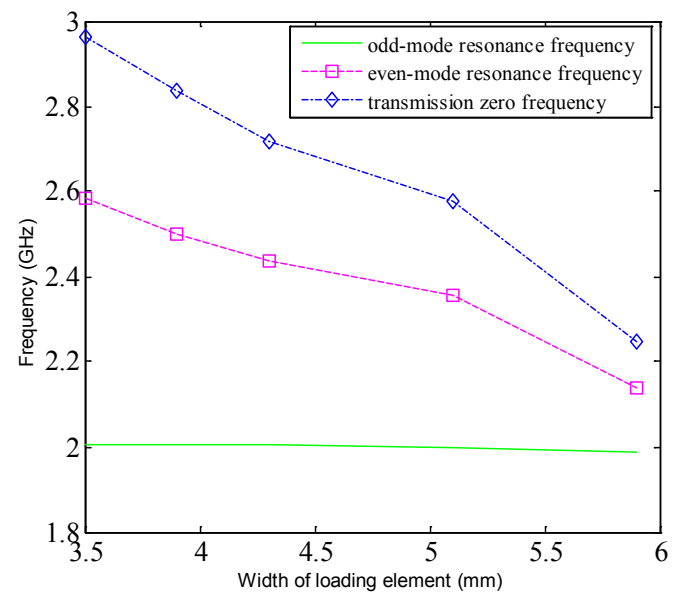

Figure 9. Width of the loading element vs. resonant frequencies variations.

\section{Simulation Results and Discussions}

\subsection{Quad-section Mixed Coupling using NRN Coupling Elements (on 2.5 \\ Substrate)}

The quad-section asymmetric response is obtained using the Teflon substrate having relative dielectric of 2.5 , substrate height of $1.5875 \mathrm{~mm}$ and loss tangent of 0.0009 in strip line technology. The metal strip is shown in the Figure 10 for the entire quad section. In Figure 11 individual section of resonator is shown. Each resonator is separated by NRN coupling element here. The port dimensions are given in Figure 12 and the NRN coupling element is shown in Figure 13 along with dimensions. At last in Figure 14 shows the frequency response of the given design in Figure 9 using EM software ${ }^{\text {15 }}$ and the response is plotted here.

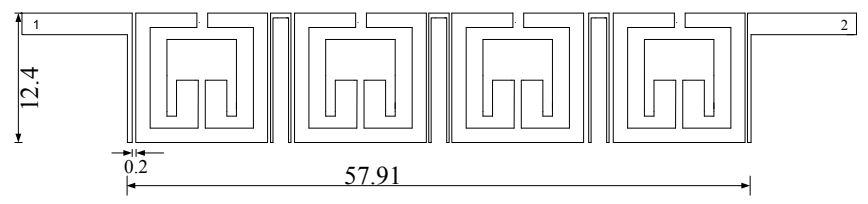

Figure 10. Quad-section using NRN coupling element.

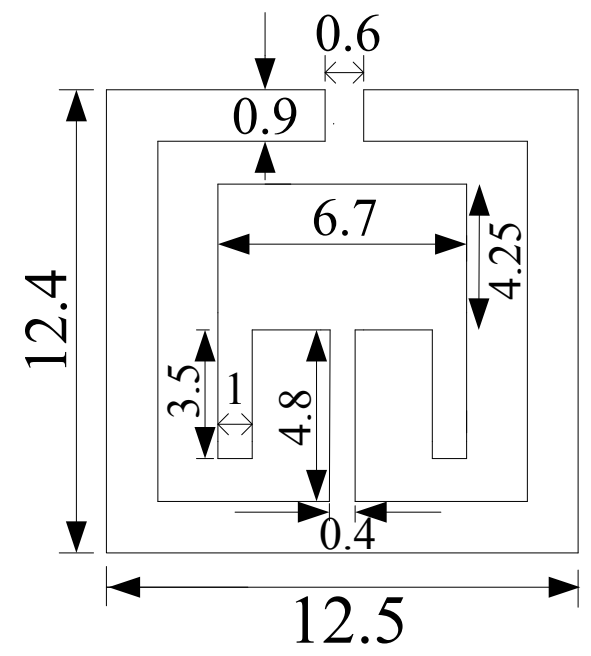

Figure 11. Single unit dimensions.

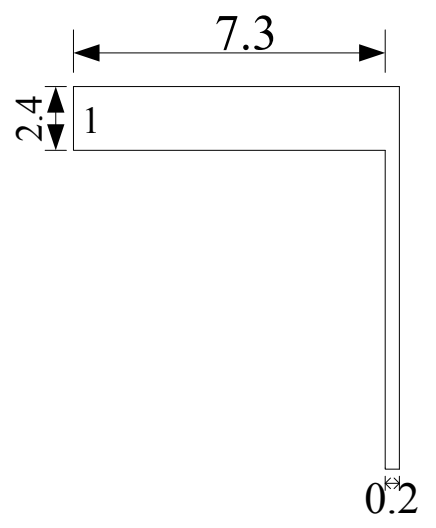

Figure 12. Port dimensions. 


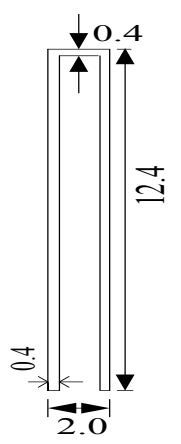

Figure 13. NRN (first and third NRN are equal length of 2 $\mathrm{mm}$, second $\mathrm{NRN}$ is $1.91 \mathrm{~mm}$ length).

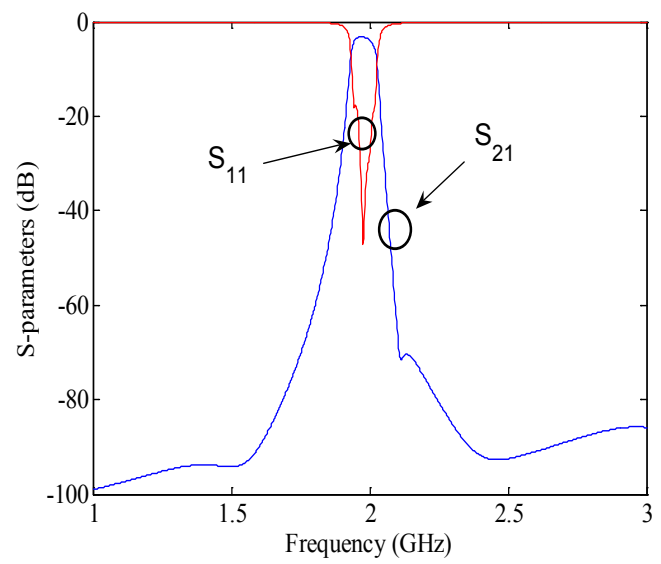

Figure 14. S-parameters of the Quad-sec using NRN coupling element.

\subsection{Quad-section Mixed Coupling using NRN Coupling Elements (on 9.2 Substrate)}

The metal strip of teflon substrate having relative dielectric of 9.2 is shown in the Figure 15 for the entire quad section. In Figure 16 individual section of resonator is shown. Each resonator is separated by NRN coupling element here. The port dimensions are given in Figure 17 and the NRN coupling element is shown in Figure 18 along with dimensions. At last in Figure 19 shows the frequency response of the given design using EM software ${ }^{17}$ and the response is plotted. After observing these two simulations it's clearly understood that the structure itself is showing greater attenuation which is not used for practical purpose. So any other method leads to decrease in the insertion loss, and maintain the remaining properties same. Since this type of Mixed coupling is leads to more loss it's better to go the other form of mixed coupling here. When the resonators are in opposite orientation then it is called mixed coupling which are separated by spacing ' $S$ ' and offset by 'd. Here two structures are proposed. Out of which one is tri-section and the other is the quad-section. These two are implemented in strip line technology on Teflon substrate.

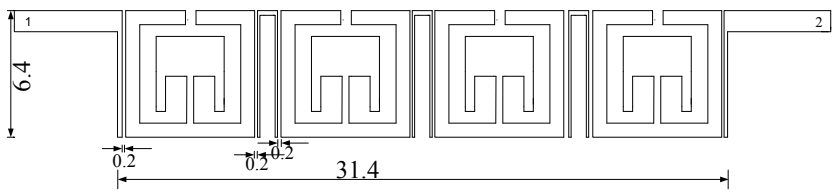

Figure 15. Quad-section using NRN coupling element.

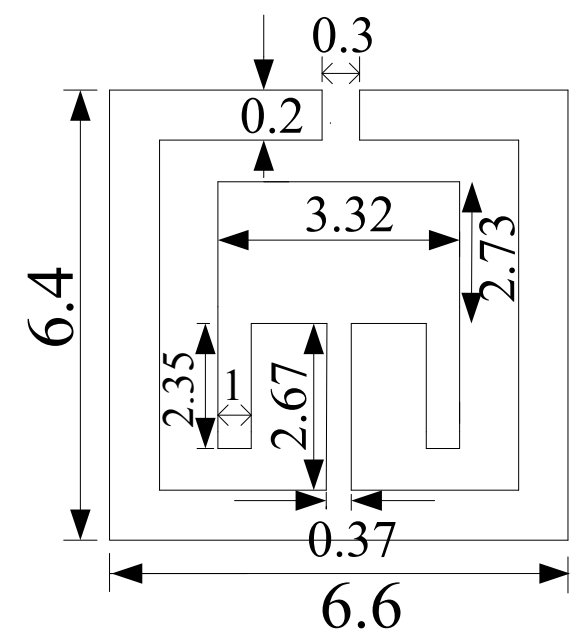

Figure 16. Single unit dimensions.

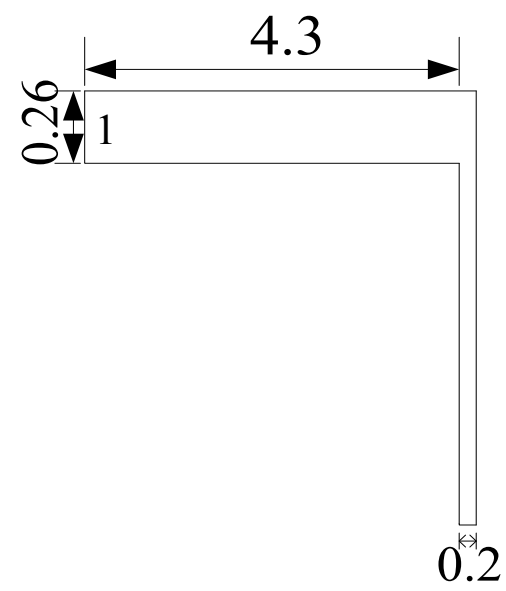

Figure 17. Port dimensions. 


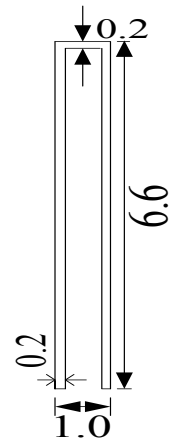

Figure 18. NRN element.

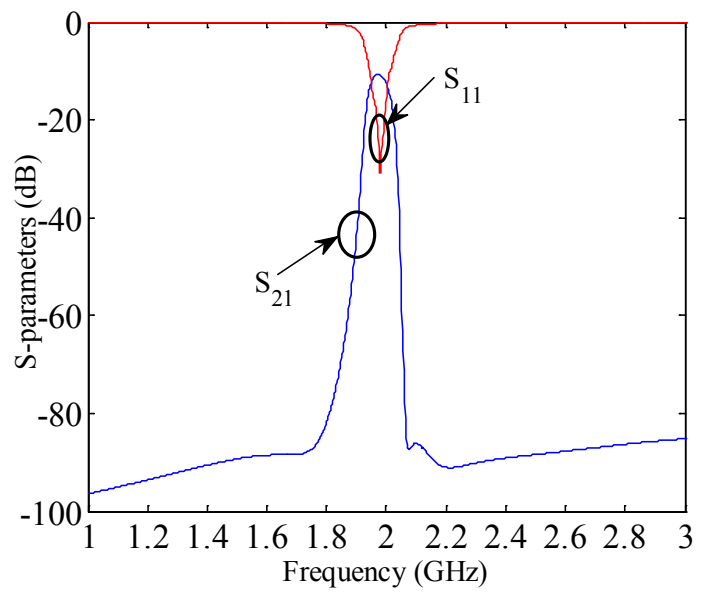

Figure 19. S-parameters of the Quad-section using NRN coupling element.

\subsection{Direct Mixed Coupling (without NRN Coupling Element)}

\subsubsection{Tri-section}

Direct Mixed Coupling (without NRN coupling element) for the entire tri section shown in Figure 20. In Figure 21 individual section of resonator is shown. Each resonator is separated by NRN coupling element here. The port dimensions are given in Figure 22 and the S-parameters of the tri-section direct mixed coupling obtained using EM software as shown in Figure 23 and the response is plotted.

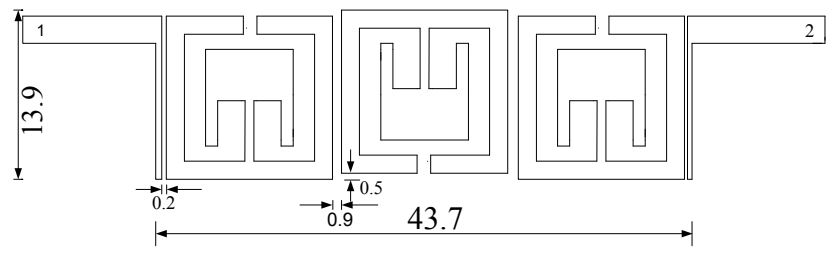

Figure 20. Tri-section direct mixed coupling.

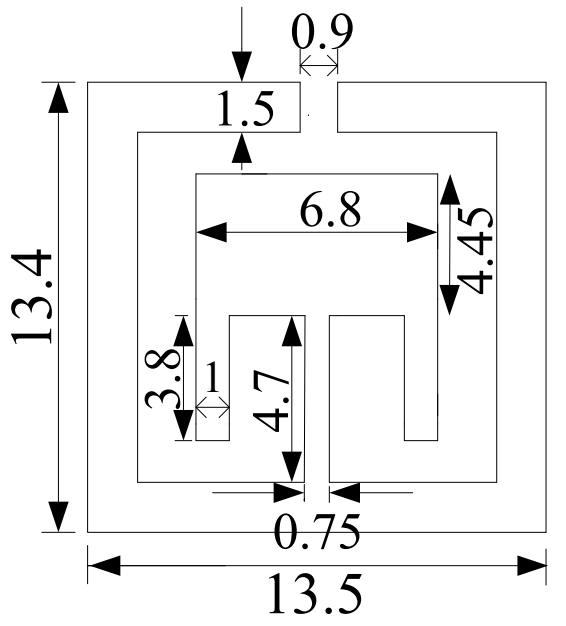

Figure 21. Single unit dimensions.

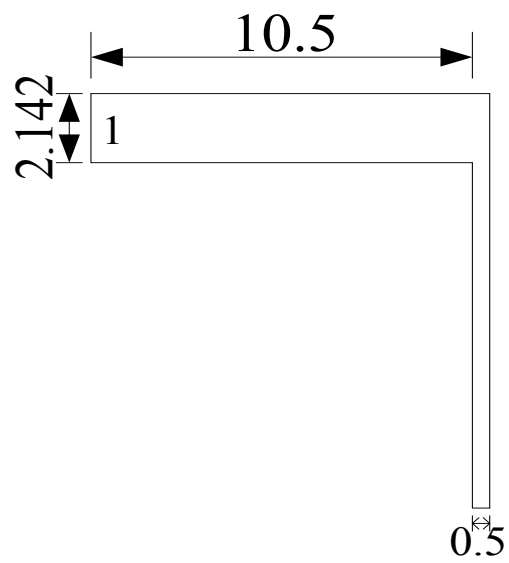

Figure 22. Port dimensions.

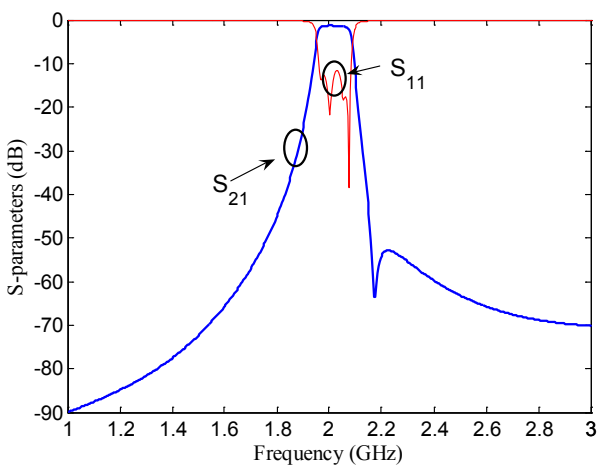

Figure 23. S-parameters of the tri-section direct mixed coupling.

The tri-section asymmetric response is obtained using the Teflon substrate having relative dielectric of 2.5, substrate height of $1.5875 \mathrm{~mm}$ and loss tangent of 0.0009 
in stripline technology. The metal strip is shown in the Figure 13 for the entire tri-section. In Figure 13individual section of resonator is shown. The port dimensions are given in Figure 13along with dimensions. At last in Figure 14 shows the frequency response of the given design in using EM software ${ }^{17}$ and the response is plotted here. The center frequency of the structure is at $2.0041 \mathrm{GHz}$, with insertion loss value of $-1.20781 \mathrm{~dB}$, the transmission zero is frequency $2.17213 \mathrm{GHz}$, with value of $-63.5681 \mathrm{~dB}$. The return loss maximum over passband is $-11.6 \mathrm{~dB}$. The flat region is over $1.9678 \mathrm{GHz}$ to $2.06852 \mathrm{GHz}$. The bandwidth of the tri-section filter is $132 \mathrm{MHz}$. The over-all tri-section size is $0.461 \lambda \mathrm{g} \times 0.1412 \lambda \mathrm{g}$.

\subsubsection{Quad-section}

Quad section direct Mixed Coupling for the entire quad section shown in Figure 24. In Figure 25 individual section of resonator is shown. Each resonator is separated by NRN coupling element here. The port dimensions are given in Figure 26 and the S-parameters of the tri-section direct mixed coupling obtained using EM software as shown in Figure 26 and the response is plotted.

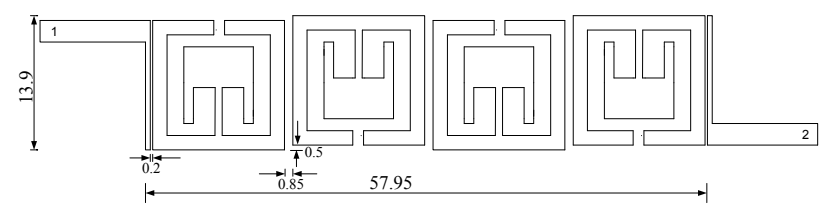

Figure 24. Quad-section direct mixed coupling.

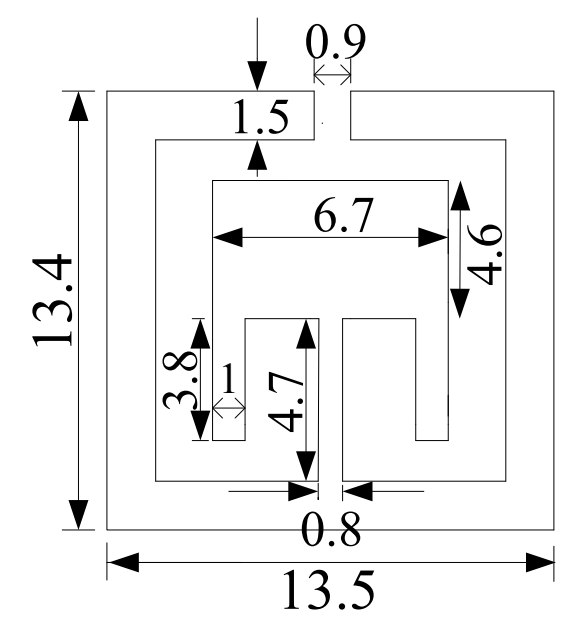

Figure 25. Single unit dimensions.

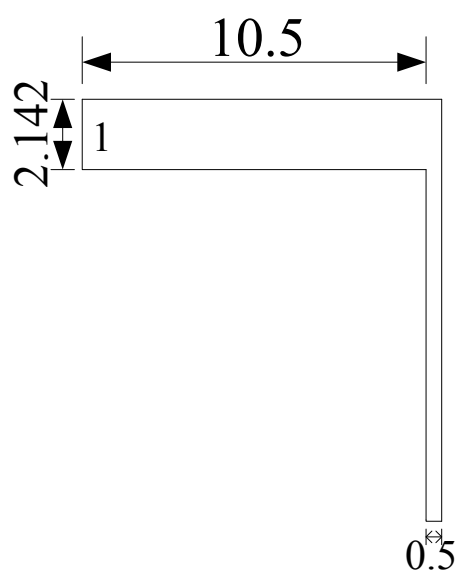

Figure 26. Port dimensions.

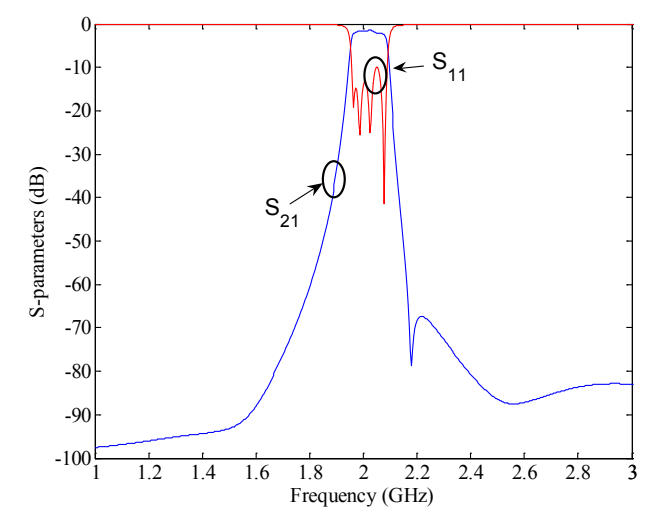

Figure 27. S-parameters of the Quad-section direct mixed coupling.

The quad-section asymmetric response is obtained using the Teflon substrate having relative dielectric of 2.5, substrate height of $1.5875 \mathrm{~mm}$ and loss tangent of 0.0009 in strip line technology. The metal strip is shown in the Figure 15 for the entire tri-section. In Figure 15 individual section of resonator is shown. The port dimensions are given in Figure 15along with dimensions. At last in Figure 16 shows the frequency response of the given design in Figure 14 using EM software 7 and the response is plotted here.

The center frequency of the structure is at $2.0246 \mathrm{GHz}$, with insertion loss value of $-1.484 \mathrm{~dB}$, the transmission zero is frequency $2.18 \mathrm{GHz}$, with insertion loss value of $-78.51 \mathrm{~dB}$. The return loss maximum over pass band is $-9.992 \mathrm{~dB}$. The flat region is over $1.9628 \mathrm{GHz}$ to 2.0702 
GHz. The bandwidth of the quad-section filter is $132 \mathrm{MHz}$. The over-all tri-section size is $0.611 \lambda \mathrm{g} \times 0.1412 \lambda \mathrm{g}$.

\section{Conclusions}

The design of compact microwave filters below $3 \mathrm{GHz}$ remains an active area of research due to great demand from the wireless communication industry within this band and comparatively large physical size of conventional resonators. Microstrip square open loop resonator suits with greater ease due to its compact size and versatility.

In this paper a compact design technique was studied which is based on the open loop resonator with a loading element. Moreover, the in-band response of the filter was not compromised by the size reduction design techniques. The tri-section and quad-section asymmetric response is obtained using the Teflon substrate having relative dielectric of 2.5 , substrate height of $1.5875 \mathrm{~mm}$ and loss tangent of 0.0009 in stripline technology. The center frequency of the structure is at $2.0246 \mathrm{GHz}$, with insertion loss value of $-1.484 \mathrm{~dB}$, the transmission zero is frequency $2.18 \mathrm{GHz}$, with insertion loss value of $-78.51 \mathrm{~dB}$. The return loss maximum over passband is $-9.992 \mathrm{~dB}$. The flat region is over $1.9628 \mathrm{GHz}$ to $2.0702 \mathrm{GHz}$. The bandwidth of the quad-section filter is $132 \mathrm{MHz}$. The over-all tri-section size is $0.611 \lambda \mathrm{g} \times 0.1412 \lambda \mathrm{g}$.

\section{References}

1. Mansour RR. Filter technologies for wireless base stations. IEEE Microwave Magazine. 2004; 5(1):68-74.

2. Hong J. Reconfigurable planar filters. IEEE Microwave Magazine. 2009;10(6):73-83.

3. Nisenoff M, Pond JM. Superconductors and microwaves. IEEE Microwave Magazine. 2009; 10(3):84-95.

4. Khanna S, Majumder D, Kumar V, Prasad S, Islam A. Impact of temperature variation on resonant frequency of active grounded inductor-based bandpass filter. Indian Journal of
Science and Technology. 2016 Aug; 9(33).DOI:10.17485/ ijst/2016/v9i33/99505.

5. Ansari MS, Ravindranath SVG, Bhatia MS, Patidar RK, Navathe CP. Application of wavelet transform for analysis of radiated electromagnetic interference in a high power terawatt laser setup. Indian Journal of Science and Technology. 2012 Nov; 5(11).DOI:10.17485/ijst/2012/v5i11/30655.

6. Plesca AT. Mechanical analysis of power electromagnetic contactors. Indian Journal of Science and Technology. 2013 Aug; 6(8).DOI:10.17485/ijst/2013/v6i8/36353.

7. Jegan G, Juliet AV, Silvia MF, Krishna GBVS. Novel design and performance analysis of rectangular frequency reconfigurable micro strip patch antenna using line feed technique for wireless applications. Indian Journal of Science and Technology. 2016 Aug; 9(29).DOI:10.17485/ijst/2016/ v9i29/98582.

8. Srikanth S, Jeyalakshmi V. Compact UWB micro strip band pass filter with open circuited stubs. Indian Journal of Science and Technology. 2015 Jul; 8(13).DOI:10.17485/ ijst/2015/v8i13/58531.

9. Hong J, Lancaster M. Canonical microstrip filter using square open-loop resonators. Electronics Letters. 1995; 31(23):2020-2.

10. LancasterMJ. Couplings of microstrip square open-loop resonators for cross-coupled planar microwave filters. IEEE Transactions on Microwave Theory and Techniques. 1996; 44(12):2099-109.

11. Kong JS.Microstrip cross-coupled trisection bandpass filters with asymmetric frequency characteristics. IEEE Proceedings on Microwaves, Antennas and Propagation. 1999; 146(1):84-90.

12. Hong JS. Design of highly selective microstrip bandpass filters with a single pair of attenuation poles at finite frequencies. IEEE Transactions on Microwave Theory and Techniques. 2000; 48(7):1098-107.

13. Sagawa M, Takahashi K, Makimoto M. Miniaturized hairpin resonator filters and their application to receiver frontend MIC's. IEEE Transactions on Microwave Theory and Techniques. 1989; 37(12):1991-97.

14. Lee S, Tsai C. New cross-coupled filter design using improved hairpin resonators. IEEE Transactions on Microwave Theory and Techniques. 2000; 48(12):2482-90.

15. IE3D Version 14.10. Zeland Software Inc., Fremont, CA; 2008. 